Alma Mater Studiorum - Università di Bologna DEPARTMENT OF ECONOMICS

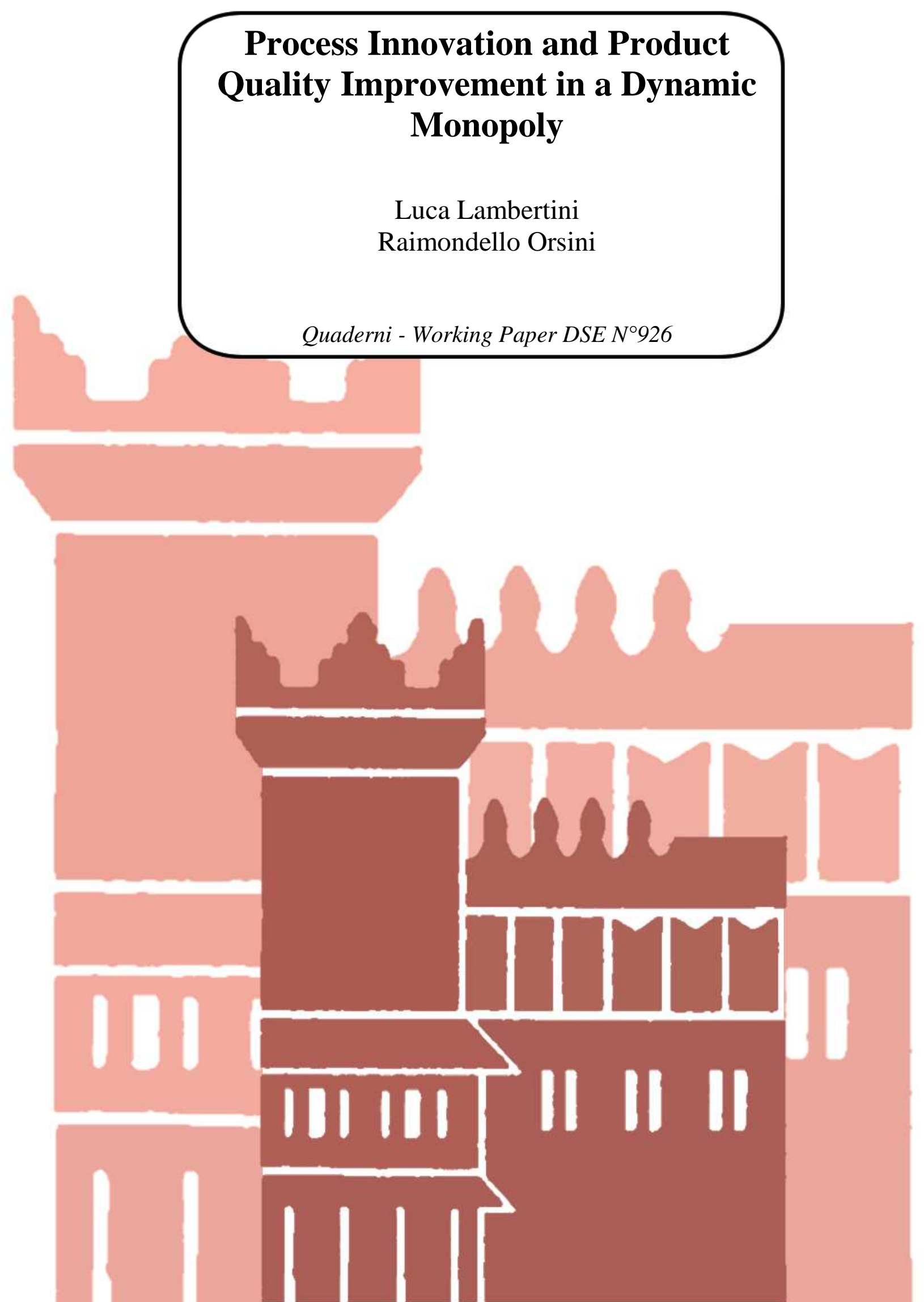




\title{
Process Innovation and Product Quality Improvement in a Dynamic Monopoly*
}

\author{
Luca Lambertini and Raimondello Orsini \\ Department of Economics, University of Bologna \\ Strada Maggiore 45, 40125 Bologna, Italy \\ luca.lambertini@unibo.it; raimondello.orsini@unibo.it
}

February 17, 2014

\begin{abstract}
We investigate the optimal R\&D portfolio of a single-product monopolist investing in cost-reducing activities accompanied by efforts improving the quality of its product. There emerges that the firm's relative incentives along the two directions are conditional upon market affluency, measured by consumers' willingness to pay for quality, and R\&D efforts are complements at equilibrium. We also perform the stability analysis, showing that a stable branch exists along the quality dimension only.
\end{abstract}

Keywords: Optimal control; Monopoly; Product quality; R\&D

JEL Codes: L12, O31

*We would like to thank Andrea Mantovani and Arsen Palestini for precious comments and suggestions. The usual disclaimer applies. 


\section{Introduction}

The impact of monopoly power on product quality is a vexata quaestio in the theory of industrial organization, at least since Spence (1975) and Mussa and Rosen (1978), where the main issue under investigation is the firm's tendency to distort quality downwards to extract as much surplus as possible from consumers' pockets. ${ }^{1}$ This literature, however, (i) is based on static models, and therefore by construction falls short of characterising the inherently dynamic nature of quality improvement; (ii) leaves out of the picture any other form of investments, such as any effort directed at decreasing marginal production costs, and finally (iii) disregards advertising activities (either persuasive or informative) aimed at increasing demand or making the product more appealing to consumers and thus convince them to pay higher prices for it.

Here, we propose a model addressing aspects (i) and (ii), ${ }^{2}$ leaving aside (iii), which has generated a lively debate since the late 1970s, stemming from the pioneering contribution of Kotowitz and Mathewson (1979). ${ }^{3}$ We characterise the optimal $R \& D$ portfolio of a monopolist investing in cost-reducing and quality improving activities while choosing the optimal output in a market populated by consumers endowed with different levels of willingness to pay for quality. Our results can be summarised as follows. First, the firm finds it optimal not to serve all consumers at equilibrium, pricing out the poorest portion of consumers. Second, we single out the conditions ensuring that both $\mathrm{R} \& \mathrm{D}$ efforts be positive at equilibrium, and we also find out that the effort directed at quality enhancement is higher than that reduc-

\footnotetext{
${ }^{1}$ This aspect has been largely debated: see Itoh (1983), Maskin and Riley (1984), Besanko, Donnenfeld and White (1987), Champsaur and Rochet (1989) and Lambertini and Orsini (2000), among others.

${ }^{2}$ The simultaneous presence of product and process innovations and their relation to product life cycle in monopoly and oligopoly models is in Lambertini and Mantovani (2009, 2010), where a different model is used, based on the representative consumer approach as in Singh and Vives (1984).

${ }^{3}$ The bulk of the resulting literature is summarised in Feichtinger et al. (1994). For a later contribution in this vein, see Colombo and Lambertini (2003).
} 
ing marginal production cost if the market is affluent enough. Third, our analysis reveals that the two types of $\mathrm{R} \& \mathrm{D}$ are complements at the steady state equilibrium. As a corollary to the main body of the analysis, the welfare assessment shows that the firm distorts quality downwards as compared to social efficiency. Finally, we also carry out the stability analysis, showing that the steady state equilibrium is a degenerate saddle point, with the stable branch emerging on the quality improvement side of the model.

The remainder of the paper is structured as follows. Section 2 illustrates the setup. The equilibrium analysis is carried out in Section 3, while Section 4 contains the stability analysis. Concluding remarks are in Section 5.

\section{The model}

Our model is a variation on the setup introduced by Mussa and Rosen (1978) and Gabszewicz and Thisse (1979). We assume the market is supplied by a single-product monopoly selling a nondurable good of quality $q(t)>0$ at price $p(t)>0$ over continuous time $t \in[0, \infty)$. The population of consumers is of constant size $\Theta>0$, and each consumer is characterised by a level of marginal willingness to pay for quality $\theta \in[0, \Theta]$. Parameter $\theta$ can be interpreted as a proxy of income or wealth. ${ }^{4}$ The mass of consumers is uniformly distributed with density 1 over such interval. At any time $t \in$ $[0, \infty)$, each individual is assumed to buy a single unit of the good or nothing at all, whereby his net surplus is

$$
U=\theta q(t)-p(t) \geq 0
$$

if he buys, otherwise it is nil. The consumer indifferent between buying or not is indexed by $\widehat{\theta}=p(t) / q(t)$; accordingly, the instantaneous inverse demand function is

$$
p(t)=[\Theta-x(t)] q(t) .
$$

\footnotetext{
${ }^{4} \mathrm{~A}$ similar although not entirely equivalent and less frequent approach consists in modelling consumer preferences by describing explicitly their income distribution. See Shaked and Sutton (1982), inter alia.
} 
On the supply side, the instantaneous cost function borne by the firm is ${ }^{5}$

$$
C(t)=c(t) x(t)+b k^{2}(t)+s y^{2}(t)
$$

where $b$ and $s$ are positive parameters and $c(t)$ is the marginal production cost of output, which can be decreased (generating thus what is usually defined as process innovation) via an $R \& D$ effort $y(t)$. The remaining variable $k(t)$ measures the instantaneous effort for product innovation (or quality improvement), increasing the level of $q(t)$. The resulting state dynamics describing the evolution of $c(t)$ and $q(t)$ over time are

$$
\begin{gathered}
\frac{d q(t)}{d t} \equiv \dot{q}=k(t)-\delta c(t) \\
\frac{d c(t)}{d t} \equiv \dot{c}=-y(t)+\eta c(t)
\end{gathered}
$$

in which $\delta>0$ is the decay rate of product quality while $\eta>0$ is the obsolescence rate affecting production technology.

The monopolist's instantaneous profits are

$$
\begin{gathered}
\pi(t)=p(t) x(t)-C(t)= \\
{[(\Theta-x(t)) q(t)-c(t)] x(t)-b k^{2}(t)+s y^{2}(t)}
\end{gathered}
$$

and the firm wants to maximise the discounted profit flow

$$
\Pi(t)=\int_{0}^{\infty} \pi(t) e^{-\rho t} d t
$$

w.r.t. controls $x(t), k(t)$ and $y(t),{ }^{6}$ under the constraints posed by the state equations (4-5), initial conditions $q(0)=q_{0}>0, c(0)=c_{0} \in(0, \Theta q(0))$, and

\footnotetext{
${ }^{5}$ We are assuming that the entire R\&D activity is carried out in house by the integrated firm. For an assessment of the bearings of outsourcing on quality improvement, and the related contractual design, see El Ouardighi and Kim (2010) and El Ouardighi and Kogan (2013), inter alia.

${ }^{6}$ The alternative between price-setting and quantity-setting behaviour is of course immaterial in a monopoly model, as can be quickly checked.
} 
the appropriate transversality conditions to be specified below. It is worth observing that the initial condition on marginal cost says that it must be strictly lower than the spending capability of the richest consumer existing in this market, in order for demand to be positive at all times. Profits are discounted at the constant rate $\rho>0$.

\section{Equilibrium analysis}

The firm's current value Hamiltonian is ${ }^{7}$

$$
\mathcal{H}=e^{-\rho t}(\pi+\lambda \dot{q}+\mu \dot{c})
$$

where $\lambda=\zeta e^{\rho t}$ and $\mu=\psi e^{\rho t}$ are the costate variables (evaluated at time $t$ ) associated with $q$ and $c$, respectively. The resulting first order conditions (FOCs) on controls and costate equations are (exponential discounting is omitted for brevity):

$$
\begin{gathered}
\frac{\partial \mathcal{H}}{\partial x}=(\Theta-2 x) q-c=0 \\
\frac{\partial \mathcal{H}}{\partial k}=-2 b k+\lambda=0 \\
\frac{\partial \mathcal{H}}{\partial y}=-2 s y-\mu=0 \\
\dot{\lambda}=-\frac{\partial \mathcal{H}}{\partial q}+\rho \lambda \Leftrightarrow \\
\dot{\lambda}=(\delta+\rho) \lambda-x(\Theta-x) \\
\dot{\mu}=-\frac{\partial \mathcal{H}}{\partial c}+\rho \mu \Leftrightarrow \\
\dot{\mu}=(\rho-\eta) \mu+x .
\end{gathered}
$$

The accompanying set of transversality conditions is $\lim _{t \rightarrow \infty} \lambda q e^{-\rho t}=0$ and $\lim _{t \rightarrow \infty} \mu c e^{-\rho t}=0$.

\footnotetext{
${ }^{7}$ Henceforth, we shall omit the explicit indication of the time argument for the sake of brevity.
} 
The FOC (9) can be solved in a quasi-static way to obtain the optimal instantaneous output $x^{*}=(\Theta q-c) /(2 q)$, so that monopoly price is $p^{*}=$ $(\Theta q+c) / 2$. Before proceeding, we may formulate

Lemma 1 For any given admissible pair of states $(q, c)$, optimal output $x^{*} \in$ $(0, \Theta)$.

Proof. To prove the Lemma, it suffices to observe that $x^{*} \in(0, \Theta)$ iff $p^{*} / q \in(0, \Theta)$, with $p^{*} / q=(\Theta q+c) /(2 q)$ and $\Theta-p^{*} / q=(\Theta q-c) /(2 q)>0$ always, since $\Theta q>p^{*}>c$ in order to enable the richest consumer to buy, either at the monopoly price or, a fortiori, at a competitive price equal to marginal cost.

From (10-11), we have

$$
\lambda=2 b k ; \mu=-2 s y
$$

and the control equations

$$
\dot{k}=\frac{\dot{\lambda}}{2 b} ; \dot{y}=-\frac{\dot{\mu}}{2 s}
$$

which, using (12-14), can be rewritten as follows:

$$
\begin{gathered}
\dot{k}=\frac{c^{2}+\left[8 b k(\delta+\rho)-\Theta^{2}\right] q^{2}}{8 b q^{2}} \\
\dot{y}=\frac{c+[4 s y(\rho-\eta)-\Theta] q}{4 s q}
\end{gathered}
$$

The system composed by (4-5) and (16-17) identifies the state-control system of the dynamic problem at hand. Now we proceed as follows. First, we impose stationarity on controls, whereby $\{\dot{k}=0, \dot{y}=0\}$ yields

$$
\begin{aligned}
k^{*} & =\frac{\Theta^{2} q^{2}-c^{2}}{8 b q^{2}(\delta+\rho)} \\
y^{*} & =\frac{\Theta q-c}{4 s q(\rho-\eta)}
\end{aligned}
$$


Note that, since $\Theta^{2} q^{2}-c^{2}=(\Theta q-c)(\Theta q+c)$ and $\Theta q-c>0$ necessarily, as it must be $\Theta q>p>c$, then $k^{*}$ is strictly positive, for any admissible levels of states $q$ and $c$. As to $y^{*}$, this is positive iff $\rho>\eta$, i.e., iff the firm's impatience outweighs the rate of depreciation of technology. Therefore, in the remainder, we shall assume $\rho>\eta$. Now, given $q$ and $c$, we can compare $k^{*}$ and $y^{*}$, to see that

$$
k^{*}-y^{*}=\frac{\Theta q-c}{4 q} \cdot \frac{(\Theta q+c) s(\rho-\eta)-2 b q(\delta+\rho)}{2 b s q(\delta+\rho)(\rho-\eta)}>0
$$

for all

$$
\Theta>\frac{2 b q(\delta+\rho)-s c(\rho-\eta)}{s(\rho-\eta) q} \equiv \Theta_{k y}
$$

Relying on the above expression and Lemma 1, we can claim the following:

Proposition 2 Take $\rho>\eta>0$. For any given admissible pair of states $(q, c)$, condition $\Theta>\max \left\{\Theta_{k y}, c / q\right\}$ suffices to ensure $k^{*}>y^{*}>0$.

Proposition 2 conveys the intuitive message that the equilibrium $R \& D$ effort for quality improvement is higher than the effort exerted for process innovation if the marginal willingness to pay for quality of the richest consumer in the market is high enough (or, equivalently, if consumers' affluency is sufficiently high): richer consumers with hedonic tastes are keen on paying higher prices for superior quality levels notwithstanding that the good they are buying is being produced at a comparatively higher marginal cost, and the firm is happy to react accordingly along the two R\&D dimensions.

A supplementary discussion can be carried out about the presence of complementarity or substitutability between the two forms of innovation, in line with an existing discussion in the literature (see Lambertini, 2003, 2004; and Lin, 2004, inter alia). At first sight, judging from (18-19), one would be tempted to conclude that, in the present model, product and process innovation are independent of each other, as $\partial k^{*} / \partial y^{*}=\partial y^{*} / \partial k^{*}=0$.

This conclusion, however, in addition to being at odds with the acquired wisdom on R\&D portfolios inherited from the theory of industrial organization (cf. Bhattacharya and Mookherjee, 1986; and Dasgupta and Maskin, 
1987), predicting some form of interplay but not independence, can be swept away by observing that both states appear in (18-19), and therefore one can carry out a simple exercise to single out the nature of the influence exerted by one type of innovation on the other. This can be done by observing that

$$
\frac{\partial k^{*}}{\partial y^{*}}=\frac{\partial k^{*}}{\partial q} \cdot \frac{\partial q}{\partial y^{*}}=\frac{\partial k^{*} / \partial q}{\partial y^{*} / \partial q}
$$

where $\partial k^{*} / \partial q=c^{2} /\left[4 b q^{3}(\delta+\rho)\right]>0$ and $\partial y^{*} / \partial q=c /\left[4 s q^{2}(\rho-\eta)\right]>0$ and therefore

$$
\frac{\partial k^{*}}{\partial y^{*}}=\frac{\partial k^{*} / \partial q}{\partial y^{*} / \partial q}=\frac{s c(\rho-\eta)}{b q(\delta+\rho)}>0
$$

for all $\rho>\eta$. Analogously,

$$
\frac{\partial y^{*}}{\partial k^{*}}=\frac{\partial y^{*}}{\partial c} \cdot \frac{\partial c}{\partial k^{*}}=\frac{\partial y^{*} / \partial c}{\partial k^{*} / \partial c}
$$

where $\partial y^{*} / \partial c=-1 /[4 s q(\rho-\eta)]<0$ and $\partial k^{*} / \partial c=-c /\left[4 b q^{2}(\delta+\rho)\right]<0$ and therefore, once again

$$
\frac{\partial y^{*}}{\partial k^{*}}=\frac{1}{\partial k^{*} / \partial y^{*}}=\frac{b q(\delta+\rho)}{s c(\rho-\eta)}>0
$$

for all $\rho>\eta$. This analysis can be summarised in

Lemma 3 Process and product innovation are complements in the neighbourhood of equilibrium.

That is, provided both efforts are positive, each one boosts the other in the neighbourhood of the steady state, thereby fostering the global innovative content of the monopolist's product. The intuitive explanation of this result is that any reduction in $c$ and any increase in $q$ increase the profitability of the firm. The first implication is obvious as a lower marginal cost produces a higher profit margin, all else equal; the second can be understood noting that higher quality levels expand the gross spending capability (measured by the product $\theta q$ ) of any consumer, and therefore contribute to expanding sales by attracting additional consumers that would be unwilling to purchase lower 
qualities. In a nutshell, any additional effort in either direction increases gross profits, thereby making available additional funds to finance the other R\&D dimension. ${ }^{8}$

We can now proceed to impose stationarity on (5), which delivers the following steady state value of marginal cost:

$$
c^{*}=\frac{\Theta q}{1+4 s q(\rho-\eta) \eta}
$$

There remains to identify the steady state level of quality. To this aim, we have to impose stationarity on (4), which now writes as follows:

$$
\dot{q}=\frac{\Theta^{2} s(\rho-\eta) \eta q[1+2 s q(\rho-\eta) \eta]}{b(\rho+\delta)[1+4 s q(\rho-\eta) \eta]^{2}}-\delta q .
$$

For future reference, note that the r.h.s. of the above equation is discontinuous in correspondence of

$$
q=-\frac{1}{4 s(\rho-\eta) \eta} \equiv \widetilde{q}<0 .
$$

Solving $\dot{q}=0$, we obtain three roots, $q=0$ and

$$
q_{ \pm}=\frac{-4 b \delta(\delta+\rho)+\Theta[\Theta s(\rho-\eta) \eta \pm \sqrt{\Gamma \Psi}]}{16 b s \delta(\delta+\rho)(\rho-\eta) \eta}
$$

with $\Gamma \equiv s(\rho-\eta) \eta$ and $\Psi \equiv \Theta^{2} s(\rho-\eta) \eta+8 b \delta(\delta+\rho)$. Note that $q_{ \pm} \in \mathbb{R}$ and $q_{-}<-1 /[4 s(\rho-\eta) \eta]$ in the whole admissible range of parameters, as it can be easily ascertained. Additionally, $q=0$ is economically inadmissible, as it would imply that all consumers would be unwilling (as well as unable) to buy the good at any positive price. Hence, we are left with a single candidate, $q^{*}=q_{+}>0$ for all

$$
\Theta>\sqrt{\frac{b(\delta+\rho) \delta}{s(\rho-\eta) \eta}} \equiv \Theta_{q}>0 .
$$

\footnotetext{
${ }^{8}$ The same can be shown to apply at any $t$, using (10-13). The related calculations are omitted for brevity.
} 
Likewise, it can be easily established that $k^{*}, y^{*}, x^{*}$ and $\pi^{*}$ are positive iff $\Theta>\Theta_{q}$. The above expression must be evaluated against (21), which now can be rewritten as follows:

$$
\Theta_{k y}=\frac{2 b \eta(\delta+\rho)-s \delta(\rho-\eta)}{s(\rho-\eta) \eta}>0
$$

for all $b>s \delta(\rho-\eta) /[2 \eta(\delta+\rho)] \equiv \underline{b}$, with

$$
\begin{gathered}
\Theta_{k y}>\Theta_{q} \forall b>\frac{s \delta(\rho-\eta)}{(\delta+\rho) \eta} \equiv \bar{b} \\
\Theta_{k y} \in\left(0, \Theta_{q}\right) \forall b \in(0, \bar{b}) .
\end{gathered}
$$

The foregoing discussion can be summarised in the following Figures 1-2. Figure 1 portrays the case $\Theta>\Theta_{q}$. In this range, the dynamics of $q$, illustrated by the horizontal arrows, shows that $q_{+}$is not only positive but also stable. The vertical dashed line indicates the discountinuity at $\widetilde{q}$.

Figure 1: Dynamics of $q, \Theta>\Theta_{q}$.

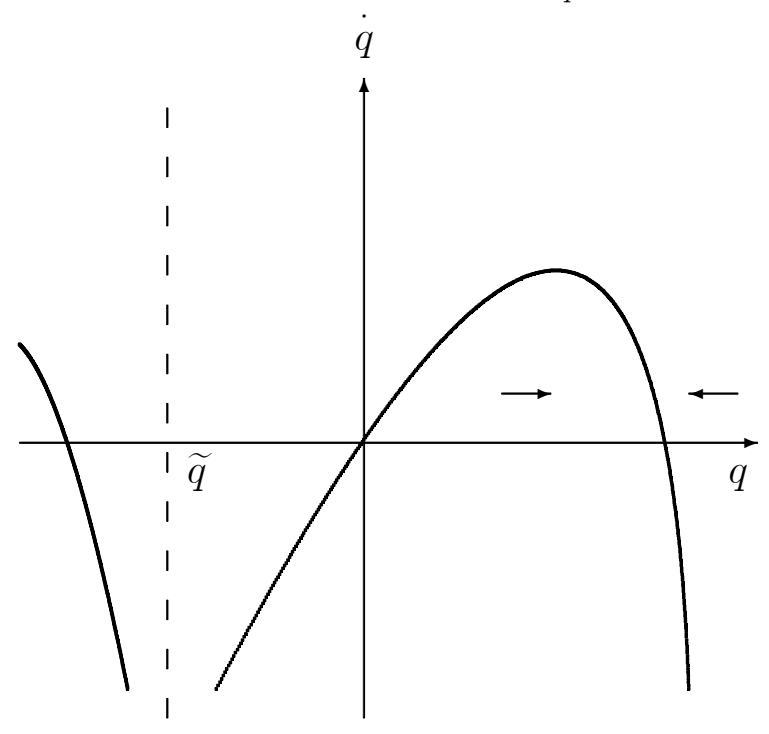

The alternative situation occurring in the parameter range identified by $\Theta \in\left(0, \Theta_{q}\right)$ can be disregarded as it is not economically meaningful: in this case, indeed, product quality drops to zero at the steady state equilibrium, which also involves that sales are nil since consumers are unwilling to buy. 
Figure 2: Equilibrium analysis in the space $\{b, \Theta\}$.

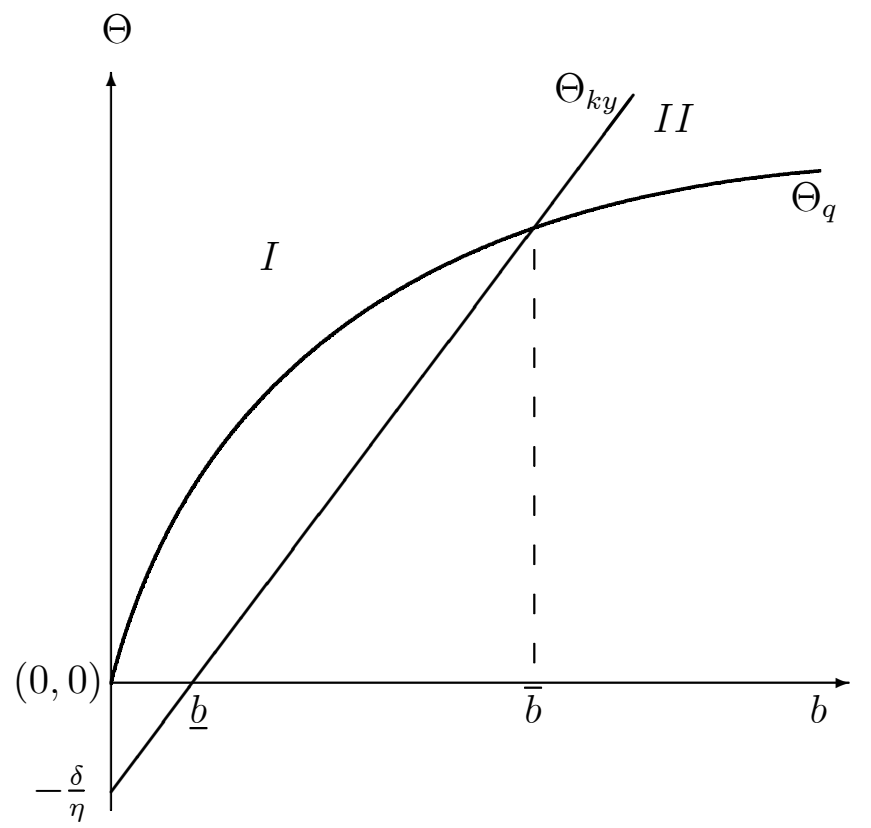

Figure 2 offers a partition of the parameter space $\{b, \Theta\}$ in which one can appreciate the bearings of market affluency and the steepness of R\&D costs for product innovation on product quality and the relative weights of process and product innovation at the steady state equilibrium.

Looking at Figure 2, we must restrict our attention to the region defined by $\Theta>\Theta_{q}$, since below the curve $\Theta_{q}$ all equilibrium magnitudes are negative. We can formulate the following:

Proposition 4 Take $\rho>\eta>0$, and consider the range $\Theta>\Theta_{q}$ :

- in area $I, \Theta>\max \left\{\Theta_{q}, \Theta_{k y}\right\}$. Here, $q^{*}=q_{+}>0$ and $k^{*}>y^{*}$;

- in area $I I, \Theta \in\left(\Theta_{q}, \Theta_{k y}\right)$. Here, $q^{*}=q_{+}>0$ but $k^{*}<y^{*}$.

The second claim appearing in the above Proposition says that, for intermediate levels of $\Theta$, although equilibrium quality is positive, the level of 
the richest consumer's marginal willingness to pay is low enough to modify the R\&D portfolio of the firm in such a way that it finds convenient to put a higher effort in process rather than in product innovation.

To complement the analysis, one can look at the welfare consequences of the firm's decision. Define social welfare as $S W=\pi+C S$, with $C S=$ $\int_{p / q}^{\Theta}(\theta q-p) d \theta$ measusing consumer surplus. Now use (18-19) and (26) to write

$$
C S\left(c^{*}, y^{*}, k^{*}\right)=\frac{2 s^{2} q^{3}(\rho-\eta)^{2} \eta^{2} \Theta^{2}}{[1+4 s q(\rho-\eta) \eta]^{2}}
$$

In correspondence of $q^{*}$, profit is maximised and therefore, if (33) is monotone w.r.t. $q$, then monopoly quality is necessarily socially inefficient in either way. It can be easily verified that (33) is monotonically increasing in $q$, which implies that the firm undersupplies product quality. This reproduces in a dynamic setup a result dating back to Spence (1975) and Mussa and Rosen (1978).

\section{$4 \quad$ Stability analysis}

The properties of the state-control system consisting of (4-5) and (16-17) must be assessed by evaluating the eigenvalues of the following $4 \times 4$ Jacobian matrix:

$$
J=\left[\begin{array}{llll}
\frac{\partial \dot{c}}{\partial c} & \frac{\partial \dot{c}}{\partial q} & \frac{\partial \dot{c}}{\partial y} & \frac{\partial \dot{c}}{\partial k} \\
\frac{\partial \dot{q}}{\partial c} & \frac{\partial \dot{q}}{\partial q} & \frac{\partial \dot{q}}{\partial y} & \frac{\partial \dot{q}}{\partial k} \\
\frac{\partial \dot{y}}{\partial c} & \frac{\partial \dot{y}}{\partial q} & \frac{\partial \dot{y}}{\partial y} & \frac{\partial \dot{y}}{\partial k} \\
\frac{\partial \dot{k}}{\partial c} & \frac{\partial \dot{k}}{\partial q} & \frac{\partial \dot{k}}{\partial y} & \frac{\partial \dot{k}}{\partial k}
\end{array}\right]=
$$




$$
\left[\begin{array}{cccc}
\eta & 0 & -1 & 0 \\
0 & -\delta & 0 & 1 \\
\frac{1}{4 s q} & -\frac{c}{4 s q^{2}} & \rho-\eta & 0 \\
\frac{c}{4 b q^{2}} & -\frac{c^{2}}{4 b q^{3}} & 0 & \delta+\rho
\end{array}\right]
$$

in correspondence of the equilibrium vector $\left\{c^{*}, q^{*}, y^{*}, k^{*}\right\}$. The analytical derivation of the eigenvalues, although possible, does not allow for a straightforward assessment of their signs as the resulting expressions are cumbersome. However, using admissible paremeter values, such as $\Theta=10$, $b=s=1 / 2, \delta=\eta=1 / 20$ and $\rho=1 / 15$, the eigenvalues can be computed numerically:

$$
\begin{gathered}
\lambda_{1}=0.1156, \lambda_{2}=-0.0489, \\
\lambda_{3}=0.0458, \lambda_{4}=0.0208
\end{gathered}
$$

The above list implies that $\left\{c^{*}, q^{*}, y^{*}, k^{*}\right\}$ is a saddle point, although a degenerate one, as it can be reached only along the branch describing the dynamic relation between quality $q$ and the product innovation control $k .^{9}$ This property can be better appreciated by splitting the stability analysis in two separate steps, each one being related to a specific state-control plane, either $\{c, y\}$ or $\{q, k\}$. In the first case, the state-control system is (5-17), whose Jacobian matrix is characterised by the following trace and determinant:

$$
\mathcal{T}(c, y)=\rho>0 ; \Delta(c, y)=\frac{1}{4 s q}+\eta(\rho-\eta)>0
$$

which implies instability. In the second case, the Jacobian matrix of (4-16) is instead characterised by

$$
\mathcal{T}(q, k)=\rho>0 ; \Delta(q, k)=\frac{c^{2}}{4 b q^{2}}-\delta(\delta+\rho)
$$

with $\Delta(q, k)<0$ for all $q>\sqrt[3]{c^{2} /[4 b \delta(\delta+\rho)]} \equiv \underline{q}$, with $q^{*}>\left.\underline{q}\right|_{c=c^{*}} \forall \Theta>$ $\Theta_{q}$. Accordingly, in the state-control plane $\{q, k\}$ we have a saddle point

\footnotetext{
${ }^{9}$ For an analogous result in a different problem concerning a differential oligopoly game, see Dragone et al. (2013, pp. 173-75).
} 
equilibrium for all $\Theta>\Theta_{q}$, i.e., in the portion of the parameter space wherein $q^{*}$ is positive and $k^{*}>y^{*}$.

\section{Concluding remarks}

We have characterised the optimal R\&D portfolio of a monopolist, showing that product improvement may indeed prevail over process innovation, if the market is sufficiently rich. When both R\&D efforts are positive, then at equilibrium they are complements, boosting each other. Equilibrium quality falls short of the socially efficient level. 


\section{References}

[1] Bhattacharya, S. and D. Mookherjee (1986). Portfolio choice in research and development. RAND Journal of Economics, 17, 594-605.

[2] Besanko, D., S. Donnenfeld \& L. White (1987). Monopoly and quality distortion: effects and remedies. Quarterly Journal of Economics, 102, 743-768.

[3] Champsaur, P. \& J.-C. Rochet (1989). Multiproduct duopolists. Econometrica, 57, 533-557.

[4] Colombo, L. \& L. Lambertini (2003). Dynamic advertising under vertical product differentiation. Journal of Optimization Theory and Applications, 119, 261-280.

[5] Dasgupta, P. and E. Maskin (1987). The simple economics of research portfolios. Economic Journal, 97, 581-595.

[6] Dragone, D., L. Lambertini \& A. Palestini (2013). The incentive to invest in environmental-friendly technologies: dynamics makes a difference, in J. Crespo Cuaresma, T. Palokangas \& A. Tarasjev (eds), Green Growth and Sustainable Development. Heidelberg, Springer, 165-188.

[7] El Ouardighi, F. \& B. Kim (2010). Supply quality management with wholesale price and revenue-sharing contracts under horizontal competition. European Journal of Operational Research, 206, 329-340.

[8] El Ouardighi, F. \& K. Kogan (2013). Dynamic conformance and design quality in a supply chain: an assessment of contracts' coordinating power, Annals of Operations Research, 211, 137-166.

[9] Feichtinger, G., R.F. Hartl \& S.P. Sethi (1994). Dynamic optimal control models in advertising: recent developments. Management Science, 40, 195-226. 
[10] Gabszewicz, J.J. \& J.-F. Thisse (1979). Price competition, quality and income disparities. Journal of Economic Theory, 20, 340-359.

[11] Itoh, M. (1983). Monopoly, product differentiation and economic welfare. Journal of Economic Theory, 31, 88-104.

[12] Kotowitz, Y. \& F. Mathewson (1979). Advertising, consumer information, and product quality. Bell Journal of Economics, 10, 566-588.

[13] Lambertini, L. (2003). The monopolist's optimal R\&D portfolio. Oxford Economic Papers, 55, 561-578.

[14] Lambertini, L. (2004). Process and product R\&D by a multiproduct monopolist: a reply to Lin. Oxford Economic Papers, 56, 745-749.

[15] Lambertini, L. \& A. Mantovani (2009). Process and product innovation by a multiproduct monopolist: a dynamic approach. International Journal of Industrial Organization, 27, 508-518.

[16] Lambertini, L. \& A. Mantovani (2010). Process and product innovation: a differential game approach to product life cycle. International Journal of Economic Theory, 6, 227-252.

[17] Lambertini, L. \& R. Orsini (2000). Process and product innovation in a vertically differentiated monopoly. Economics Letters, 68, 333-337.

[18] Lin, P. (2004). Process and product R\&D by a multiproduct monopolist. Oxford Economic Papers, 56, 735-743.

[19] Maskin, E. \& J. Riley (1984). Monopoly with incomplete information, RAND Journal of Economics, 15, 171-196.

[20] Mussa, M. \& S. Rosen (1978). Monopoly and product quality. Journal of Economic Theory, 18, 301-317.

[21] Shaked, A. \& J. Sutton (1982). Relaxing price competition through product differentiation. Review of Economic Studies, 49, 3-13. 
[22] Singh, N. \& X. Vives (1984). Price and quantity competition in a differentiated duopoly. RAND Journal of Economics, 15, 546-554.

[23] Spence, A.M. (1975). Monopoly, quality and regulation. Bell Journal of Economics, 6, 417-429. 


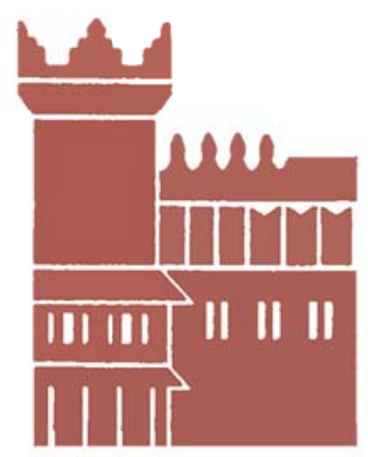

Alma Mater Studiorum - Università di Bologna DEPARTMENT OF ECONOMICS

Strada Maggiore 45

40125 Bologna - Italy

Tel. +39051 2092604

Fax +390512092664

http://www.dse.unibo.it 